\title{
A Novel Metabolic Connectome Method to Predict Progression to Mild Cognitive Impairment
}

\author{
Min Wang, ${ }^{1}$ Zhuangzhi Yan $\mathbb{D}^{1},{ }^{1}$ Shu-yun Xiao $\mathbb{D}^{2},{ }^{2}$ Chuantao Zuo, ${ }^{3}$ and Jiehui Jiang ${ }^{1,4}$ \\ ${ }^{1}$ Institute of Biomedical Engineering, School of Communication and Information Engineering, Shanghai University, Shanghai, China \\ ${ }^{2}$ Department of Brain and Mental Disease, Shanghai Hospital of Traditional Chinese Medicine, Shanghai, China \\ ${ }^{3}$ PET Center, Huashan Hospital, Fudan University, Shanghai, China \\ ${ }^{4}$ Shanghai Institute for Advanced Communication and Data Science, Shanghai University, Shanghai, China
}

Correspondence should be addressed to Zhuangzhi Yan; zzyan@shu.edu.cn and Shu-yun Xiao; lindaxsy@163.com

Received 16 June 2020; Revised 17 July 2020; Accepted 10 August 2020; Published 18 August 2020

Academic Editor: Muh-Shi Lin

Copyright (c) 2020 Min Wang et al. This is an open access article distributed under the Creative Commons Attribution License, which permits unrestricted use, distribution, and reproduction in any medium, provided the original work is properly cited.

\begin{abstract}
Objective. Glucose-based positron emission tomography (PET) imaging has been widely used to predict the progression of mild cognitive impairment (MCI) into Alzheimer's disease (AD) clinically. However, existing discriminant methods are unsubtle to reveal pathophysiological changes. Therefore, we present a novel metabolic connectome-based predictive modeling to predict progression from MCI to AD accurately. Methods. In this study, we acquired fluorodeoxyglucose PET images and clinical assessments from $420 \mathrm{MCI}$ patients with 36 months follow-up. Individual metabolic network based on connectome analysis was constructed, and the metabolic connectivity in this network was extracted as predictive features. Three different classification strategies were implemented to interrogate the predictive performance. To verify the effectivity of selected features, specific brain regions associated with MCI conversion were identified based on these features and compared with prior knowledge. Results. As a result, 4005 connectome features were obtained, and 153 in which were selected as efficient features. Our proposed feature extraction method had achieved $85.2 \%$ accuracy for MCI conversion prediction (sensitivity: 88.1\%; specificity: $81.2 \%$; and AUC: 0.933). The discriminative brain regions associated with MCI conversion were mainly located in the precentral gyrus, precuneus, lingual, and inferior frontal gyrus. Conclusion. Overall, the results suggest that our proposed individual metabolic connectome method has great potential to predict whether MCI patients will progress to AD. The metabolic connectome may help to identify brain metabolic dysfunction and build a clinically applicable biomarker to predict the MCI progression.
\end{abstract}

\section{Introduction}

Alzheimer's disease (AD) is a neurodegenerative brain disease and the most common cause of dementia, affecting millions of individuals worldwide [1]. In the intermediate stage between healthy aging and $\mathrm{AD}$, one had developed cognitive deficits that can be diagnosed as mild cognitive impairment (MCI) [2]. Yet MCI disease is very complex, manifesting in clinical and neuropathological heterogeneity. The development of MCI is so labile that some remain in stable stage for many years after diagnosis and even revert to normal cognition. Early diagnosis of whether MCI patients will progress into $\mathrm{AD}$ is a daunting challenge.

Currently, most diagnosis studies on MCI conversion are using neuroimaging to acquire the features among MCI groups and then consider these features as biomarkers to predict MCI progression. As a frequently used neuroimaging technique in clinics, ${ }^{18} \mathrm{~F}$-fluorodeoxyglucose (FDG) positron emission tomography (PET) imaging has been employed to detect the progression from MCI to $\mathrm{AD}$ [3]. For instance, clinical studies have revealed that FDG-PET could capture the information of resting-state regional cerebral glucose metabolic rate and predicted progression from $\mathrm{MCI}$ to $\mathrm{AD}$, and it is mainly due to FDG uptake is reduced in abnormal high cerebrospinal fluid amyloid- $\beta$ concentrations [4-6]. Recently, as the studies for FDG-PET imaging has advanced, there are various predictive modeling that has been proposed. Previously, some studies had used voxel-wise or region-of-interest- (ROI-) wise quantitative metabolic measures to predict MCI patient's progress into AD [7-9]. 


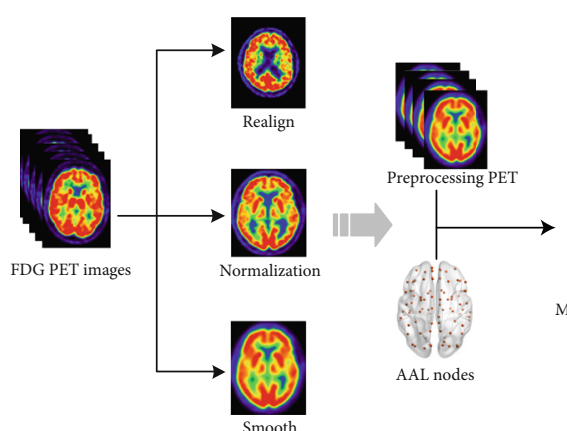

Preprocessing

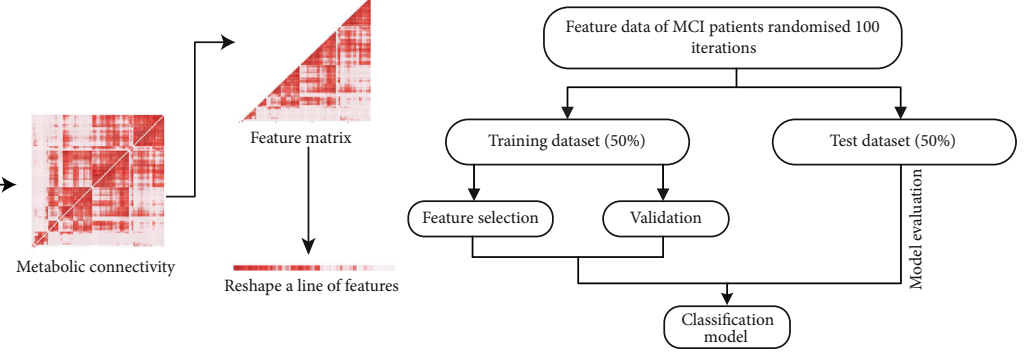

Connectome-based predictive modeling

FIGURE 1: The overall framework of the proposed metabolic connectome-based predictive modeling (CPM) approach in this study.

However, the subtle difference between stable $\mathrm{MCI}$ and progression MCI causes it difficult to diagnose and require exquisite predictive modeling. Besides, some studies have explored the application of deep learning methods, such as convolutional neural network (CNN) and deep Boltzmann machine (DBM) [10-12]. Nonetheless, deep learning methods face the challenge that limited sample of FDGPET images and inaccessible biomarkers could hardly reveal the neuropathological changes associated with MCI conversion. Specifically, a previous study evaluated different predictive models on the same FDG-PET images for reproducible evaluation among MCI patients, and the results showed that the accuracy of classification range between $67 \%$ and $83 \%$ [13]. Thus, the development of new predictive modeling is necessary to assist diagnosis in clinical assessments and provide higher performance to predict MCI progression.

Connectome-based predictive modeling (CPM) is a novel data-driven protocol for developing predictive models of brain-behavior relationships, which has addressed challenges in brain neurodegenerative disease $[14,15]$. Brain connectivity characterizes by different brain regions and the relationship between paired regions and discloses the dynamic communication by neuronal activity. The brain network based on FDG-PET images is an exciting new opportunity to understand the neurological disorders and has been proved adept at analyzing the abnormalities in $\mathrm{AD}$ patients [16-18]. As a result, CPM has the potential to provide a novel predictive model for MCI conversion.

The aim of this study is therefore to combine CPM with glucose metabolic imaging to identify discriminative features for accurately classifying whether MCI patients will progress to AD. Specifically, we have two secondary aims: (1) verify whether CPM can be used as a novel feature extraction method in FDG-PET images and (2) evaluate the predictive performance of our proposed metabolic CPM in MCI groups.

\section{Materials and Methods}

2.1. Motivation. The goal of this study is to develop a predictive model to capture predictive individual differences in MCI patients using CPM and FDG-PET imaging. We hypothesize that the combination of CPM and glucose metabolic imaging may identify the subtle brain metabolic dys- function and use this information to obtain remarkable diagnosis performance. The framework of our proposed approach is summarized in Figure 1.

2.2. Participants and Imaging Protocols. The ${ }^{18} \mathrm{~F}-\mathrm{FDG}-\mathrm{PET}$ data used in this study were obtained from the Alzheimer's Disease Neuroimaging Initiative (ADNI) database (http:// adni.loni.usc.edu). The primary goal of ADNI has been to test whether serial magnetic resonance imaging (MRI), positron emission tomography (PET), other biological markers, and clinical and neuropsychological assessments can be combined to measure the progression of mild cognitive impairment (MCI) and early Alzheimer's disease (AD). The institutional review board of ADNI approved all aspects of this study, and each participant has given written informed consent to undergo PET scanning of a long-term observational study.

This study acquired 420 MCI participants with FDGPET scanning from ADNI-1, ADNI-2, and ADNI-GO database. The participant group was comprised of 242 stable MCI (sMCI) subjects and 178 progressive MCI (pMCI) subjects, and the images were used to establish a predictive model and test the validity of the model. The detailed eligibility criteria for all participants included the following: all participants underwent FDG-PET scanning and clinical cognitive evaluations at baseline visit and were followed during at least 36 months; stable MCI participants were diagnosed of MCI at baseline visit and did not progress to $\mathrm{AD}$ within 36 months of follow-up; and progressive MCI participants were diagnosed of $\mathrm{MCI}$ at baseline visit and progressed to $\mathrm{AD}$ within 36 months of follow-up. The demographic and clinical characteristics of all participants are summarized in Table 1.

Resting-state FDG-PET images at baseline visits were acquired and the detailed acquisition process could be found in the online information of ADNI. All participants were scanned using ${ }^{18} \mathrm{~F}$-fluorodeoxyglucose (FDG) positron emission tomography (PET). There were 218 dynamic 3D images with six $5 \mathrm{~min}$ frames acquired $30 \mathrm{~min}$ after injection of 185 $\pm 18.5 \mathrm{MBq}$ FDG. Besides, 202 participants were scanned with a static 30 -minute acquisition.

2.3. Preprocessing. We preprocessed the brain FDG-PET images using the Statistical Parametric Mapping software (SPM12; Wellcome Department of Imaging Neuroscience, 
TABLE 1: Demographic information of all participants.

\begin{tabular}{lccc}
\hline Group & sMCI $(n=242$ & pMCI $(n=178$ & $P$ value \\
\hline Sex (M/F) & $136 / 106$ & $102 / 76$ & $0.89^{\mathrm{a}}$ \\
Age (year) & $71.6 \pm 7.82$ & $73.7 \pm 6.91$ & $0.004^{\mathrm{b}}$ \\
Education (year) & $15.9 \pm 2.67$ & $16.1 \pm 2.65$ & $0.496^{\mathrm{c}}$ \\
MMSE & $28.2 \pm 1.64$ & $27.1 \pm 1.80$ & $<0.001^{\mathrm{b}}$ \\
CDRSB & $1(0.5,1.5)$ & $1.5(1,2.5)$ & $<0.001^{\mathrm{c}}$ \\
ADAS11 & $8.4 \pm 3.4$ & $13.0 \pm 4.6$ & $<0.001^{\mathrm{b}}$ \\
ADAS13 & $13.5 \pm 5.5$ & $21.0 \pm 6.2$ & $<0.001^{\mathrm{b}}$ \\
Conversion time & $\backslash$ & $20.6 \pm 10.3$ & $\backslash$ \\
(month) & $44.6 \%$ & $68.5 \%$ & $<0.001^{\mathrm{a}}$ \\
APOE $\varepsilon 4$ positive rate & & & \\
\hline
\end{tabular}

Data are given as mean \pm standard deviation. $P^{\mathrm{a}}$ : the chi-square test; $P^{\mathrm{b}}$ : the two-sample $t$-test; $P^{c}$ : the Wilcoxon rank-sum test. MMSE: Mini-Mental State Examination; CDRSB: Clinical Dementia Rating Sum of Boxes; ADAS11: The Alzheimer's Disease Assessment Scale with 11 tasks; ADAS13: The Alzheimer's Disease Assessment Scale with 13 tasks; APOE $\varepsilon 4$ positive rate: positive or negative for the presence of at least one $\varepsilon 4$ allele.

Institute of Neurology, London, UK) implemented in MATLAB (MathWorks Inc., Sherborn, MA). We realigned a time-series of FDG-PET images to generate a stable FDGPET image. PET images were then spatially normalized into the Montreal Neurological Institute (MNI) brain space with linear and nonlinear $3 \mathrm{D}$ transformations. The normalized PET images were smoothed by a Gaussian filter of $8 \mathrm{~mm}$ full width at half maximum (FWHM) over a 3D space to blur individual variations in gyral anatomy and to increase signal to noise ratio for statistical analysis. Each PET image was intensity normalized to the global mean brain uptake to avoid individual uptake differences. For further analysis, the whole brain images were divided into 90 regions-of-interest (ROIs) defined by the automated anatomical labeling (AAL) atlas [19].

2.4. Metabolic Connectome Analysis. To acquire individual metabolic network from the FDG-PET image, we employed an individual-level graphical approach for metabolic connectivity, namely the Kullback-Leibler Divergence Similarity Estimation (KLSE) [20]. The globally normalized metabolic activity in ROIs was used to generate a glucose metabolic network for each participant. Firstly, the 90 cortical and subcortical ROIs derived by AAL were defined as network nodes. Then, for estimating the metabolic connectivity (metabolic correlations) between network nodes, we applied relative entropy into the spatial dimension, where the FDG-PET signal in ROIs reflected afferent synaptic activity and probability distribution between these ROIs denote interneuronal information transfer. The closer the relative entropy was to zero, the stronger metabolic connectivity between two random ROIs.

The detailed mathematical derivation of metabolic connectivity included the following three steps (see Figure 2). Firstly, we estimated the probability density function (PDF) of a random brain region (ROI) using a nonparametric way, namely the kernel density estimation (KDE). The esti- mation of kernel width was using a solve-the-equation bandwidth. Given the sample array quantifying the metabolic intensity of each voxel with ROI, we estimated the characteristic function as

$$
\widehat{\varphi}(\mathrm{t})=\left(\frac{1}{n} \sum_{j=1}^{n} e^{i t x_{j}}\right) .
$$

In this study, we had chosen the Gaussian function as a damping function to circumvent the question of diverging integral. After the damping function has been chosen, the Fourier transform formula may be applied, and the density estimation can be derived. Secondly, we assessed the metabolic correlation using the relative entropy between ROIs, which was estimated from the symmetric Kullback-Leibler (KL) divergence. The similarity of pairwise probability density functions (PDFs) was measured as given in the below mathematical equation:

$$
D_{K L}(P \| Q)=\int_{X}\left(P(x) \log \frac{P(x)}{Q(x)}+Q(x) \log \frac{Q(x)}{P(x)}\right) d x .
$$

in which $P$ and $Q$ represent the probability density functions PDFs of voxel intensities in pairwise ROIs. Lastly, t4he normalized similarity of these ROIs was acquired by KL divergence using the following representation:

$$
K L S(P \| Q)=\exp \left(-D_{K L}(P \| Q)\right) .
$$

Thus, a metabolic correlation matrix $(90 \times 90$, region $\times$ region, 90 is the number of ROI) for each participant was obtained by the magnitude of KL-based similarity (KLS), where the correlation matrix elements represented the metabolic connectivity between pairwise nodes. Thus, we had constructed a metabolic network for each subject by AAL template (nodes) and KLSE algorithm (metabolic connectivity).

2.5. Predictive Modeling Analysis. For the metabolic network of each participant, a feature vector was obtained by extracting the lower triangular elements of correlation matrix. Each participant could acquire $4005(90 \times 89 / 2)$ features which defined as the metabolic connectivity between ROIs. The feature vectors then underwent predictive modeling analysis to discriminate sMCI and pMCI groups. To evaluate our proposed metabolic CPM approach fairly and minimize the influence factors, we employed three different classifiers, that are support vector machine (SVM), logistic regression (LR) model, and random forest (RF). Meanwhile, we also performed the Hosmer-Lemeshow goodness of fit test for the LR model.

To avoid the sampling variability of training and test datasets and obtain more stable estimates of predictive performance, we implemented the randomized crossvalidation strategy. The detailed cross-validation procedures include two main steps. Firstly, all MCI participants were randomly partitioned into training dataset $(50 \%$, training the model) and test dataset $(50 \%$, test 


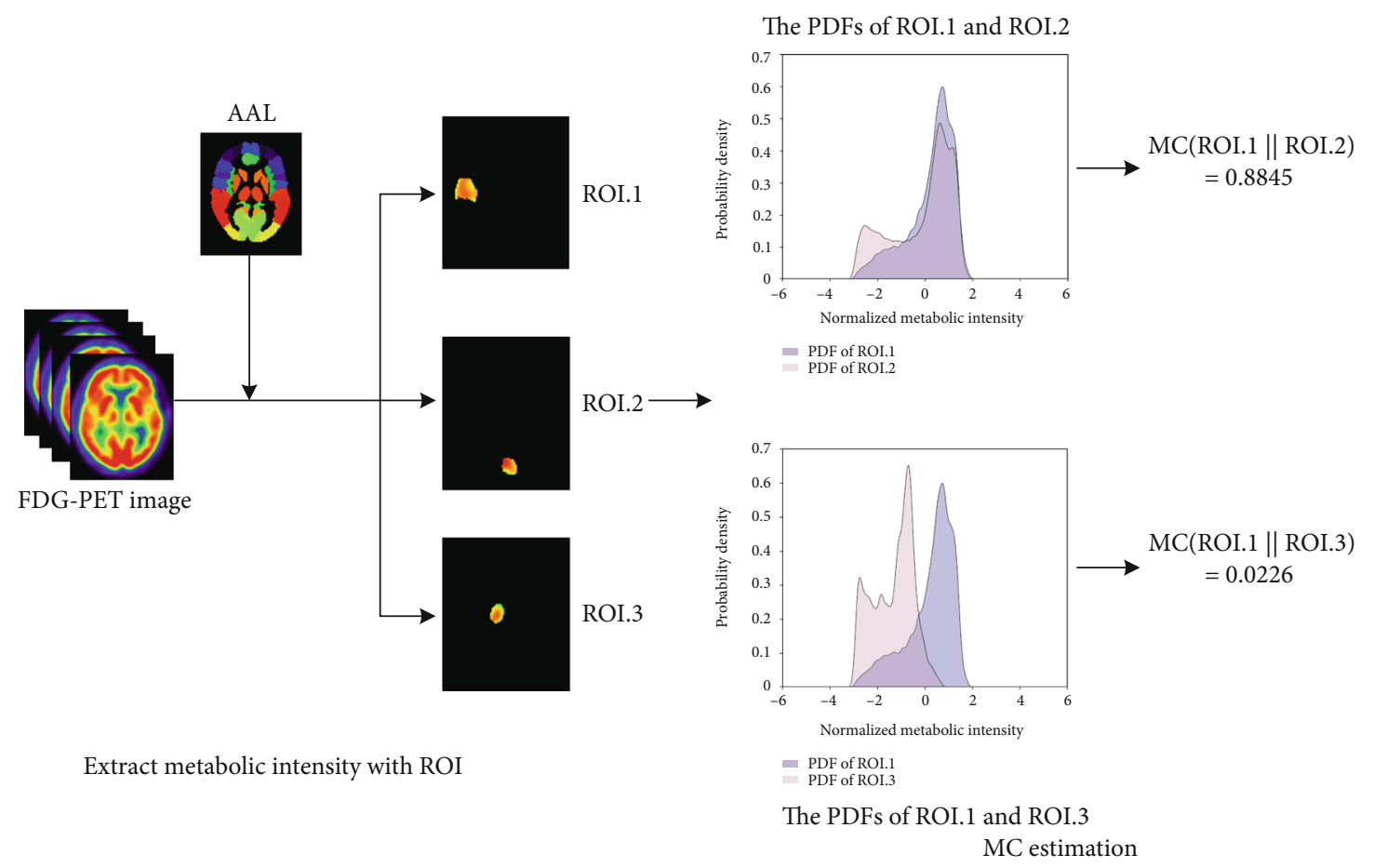

FIGURE 2: The framework for the estimation of metabolic connectivity (MC) between pairwise regions from individual FDG-PET image. Firstly, the FDG-PET image was divided into 90 ROIs, and the metabolic intensity values of voxels with random ROI were extracted. Then, kernel density estimation was employed to estimate the probability density function (PDF) of each ROI. Lastly, the KLSE algorithm was implemented to measure the metabolic correlation by the similarity among PDFs.

classification performance) for multiple times (100 iterations). Secondly, we implemented 10 -fold cross-validation in the training dataset for hyperparameter optimization. In each random sampling of the training dataset, sparse regression least absolute shrinkage and selection operator (LASSO) approach was adopted to reduce the redundant features and to select the feature subset with higher discriminability [21]. The fitting models were built using those selected features. The predictive performance of models was evaluated by accuracy, sensitivity, specificity, the receiver-operating-characteristic curve, and the relevant area under the curve (AUC). The random split procedure was repeated for 100 iterations, and the mean and standard deviation of classification indicators were reported. SVM with linear kernel, L1-penalized LR model, random forest, and following receiver-operatingcharacteristic curve analysis and accuracy measurements were performed using the LIBSVM 3.23 toolbox and Statistics and Machine Learning Toolbox implemented in MATLAB.

2.6. Comparative Experiment. To further evaluate the performance of our proposed approach, two previous predictive methods in FDG-PET imaging were applied to the same predictive tasks: (1) the conventional feature quantification approach was performed based on mean metabolic uptakes in brain regions, and the FDG uptake values were regarded as features; (2) the spatial covariance analysis was performed on the training dataset to acquire a metabolic $\mathrm{AD}$ conversion-related pattern (ADCRP) topography, in which each voxel value represented the predictive weights [22]. The ADCRP expressions of each FDG-PET images were obtained by the $Z$-transformed score and regarded as the input features of the model. The comparative experiments underwent the same process procedures with the above metabolic connectivity.

2.7. Structural Brain Regions Associated with Effective Features. To verify the effectiveness of our proposed method, we further identified the structural brain regions associated with effective features. Firstly, part connectome features were considered efficient and discriminative features after undergoing the above feature select procedures. Then, the correlation matrix was generated which included only selected connectome features, and the weights of 90 brain regions were derived by column-summing the entries. In this case, the higher weight of regions implied that the region had more discriminative connectivity incident upon it. Lastly, we normalized the region weights by $Z$-score and sorted by the sign of the corresponding region weights. We obtained the structural brain regions associated with effective features by the criteria: $Z$-scored weight of region was greater than +1.0 . These brain regions were considered relevant to $\mathrm{AD}$ progression [23].

\section{Result}

3.1. Clinical Characteristics. Clinical and demographic characteristics are reported in Table 1 . The result of the age $(P=0.004)$ and the APOE $\varepsilon 4$ positive rate $(P<0.001)$ 


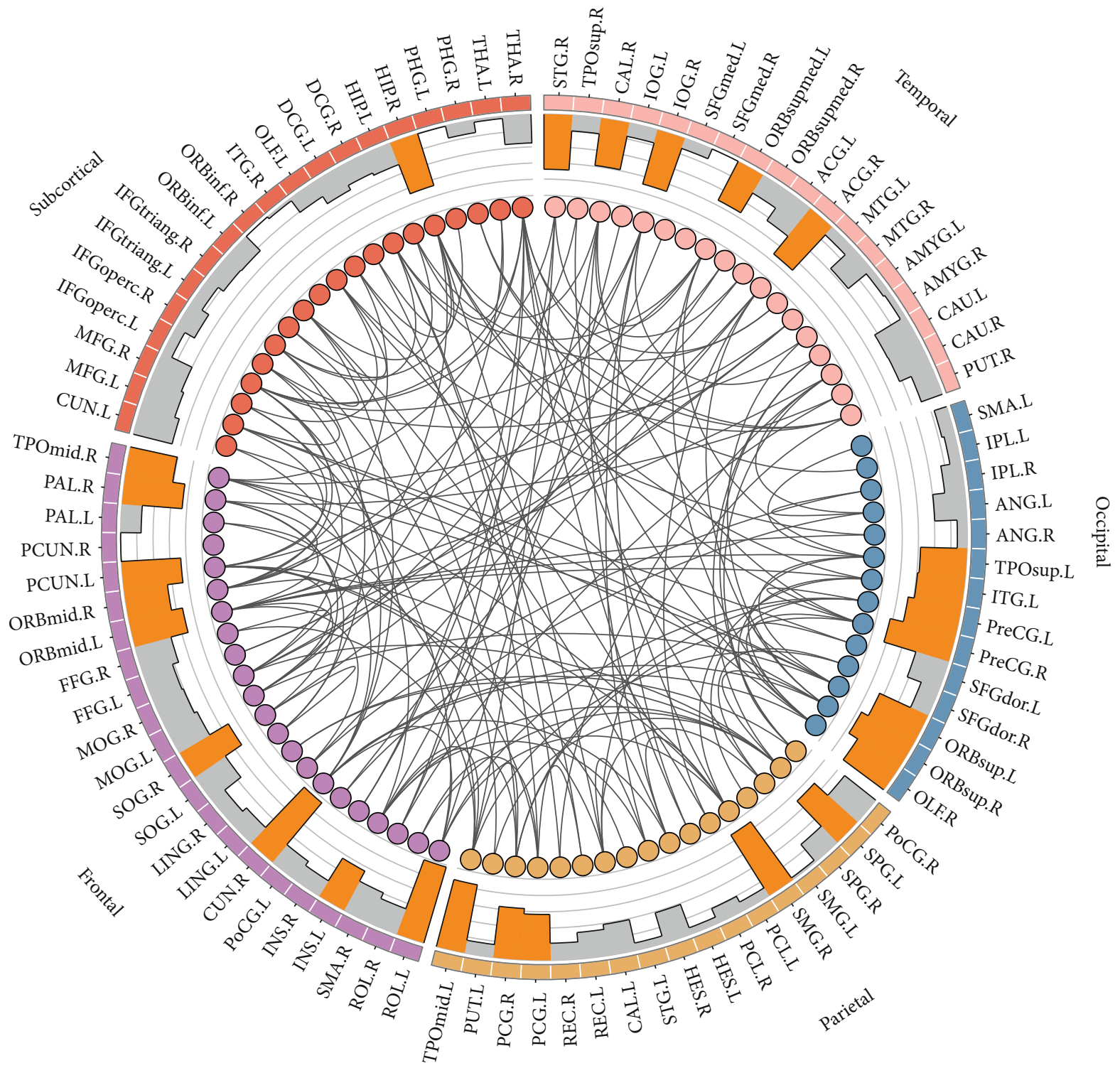

FIgURE 3: The brain metabolic connectivity associated with MCI conversion. The nodes in the circle represent 90 brain regions (ROIs). The different colors of nodes on the circle represent different anatomical classifications of brain regions (purple: frontal, orange: parietal, blue: occipital, pink: temporal, and red: subcortical). The black lines within nodes represent functional connectivity between ROIs selected with sufficient discriminative ability and associated with AD progression. The yellow histogram bars represent the hubs with more metabolic connectivity and the grey bars with little discriminative ability.

showed significant changes between sMCI and pMCI groups. The cognitive assessments in the pMCI subjects tended to decrease than that in the sMCI subjects (Mini-Mental State Examination (MMSE), $P<0.001$; Clinical Dementia Rating Sum of Boxes (CDRSB), $P<0.001$; The Alzheimer's Disease Assessment Scale with 11 tasks (ADAS11), $P<0.001$; and The Alzheimer's Disease Assessment Scale with 13 tasks (ADAS 13), $P<0.001$ ). There were no significant differences in sex $(P=0.89)$ or education level $(P=0.496)$.

3.2. Predictive Modeling Analysis. The 4005 functional connectivities drawn from the KLSE algorithm were the input features for predictive analysis. In the randomized crossvalidations in 100 iterations, 153 metabolic connectivities were selected. Figure 3 shows the brain metabolic connectivity associated with MCI conversion. The hubs were defined as the network nodes that had more quantitatively selected features. These hubs were highlighted to be associated with MCI conversion and mainly located in the brain regions including the precentral gyrus, precuneus, lingual, and inferior frontal gyrus.

We compared the predictive performances of different feature extraction methods: (1) ROI uptake; (2) MCI pattern; and (3) metabolic CPM. Table 2 shows the results of different methods using three classifiers, and the best results for MCI groups diagnosis were achieved by the selected features from connectome with SVM classifier. The corresponding accuracy, sensitivity, specificity, and AUC values were $85.2 \%$, 
TABLE 2: Predictive performance of different methods among MCI groups.

\begin{tabular}{|c|c|c|c|c|c|}
\hline Classifier & Predictive method & Accuracy (\%) & Sensitivity (\%) & Specificity (\%) & AUC \\
\hline \multirow{3}{*}{ SVM } & ROI uptake & $74.8 \pm 2.41$ & $82.2 \pm 1.72$ & $66.7 \pm 2.51$ & $0.829 \pm 0.035$ \\
\hline & MCI pattern & $76.7 \pm 2.48$ & $83.7 \pm 4.46$ & $67.1 \pm 6.29$ & $0.831 \pm 0.026$ \\
\hline & Connectome & $85.2 \pm 2.34$ & $88.1 \pm 3.17$ & $81.2 \pm 4.28$ & $0.933 \pm 0.014$ \\
\hline \multirow{3}{*}{ LR model } & ROI uptake & $72.4 \pm 2.73$ & $81.1 \pm 5.99$ & $60.7 \pm 4.83$ & $0.748 \pm 0.037$ \\
\hline & MCI pattern & $74.8 \pm 4.36$ & $82.3 \pm 2.49$ & $66.8 \pm 5.91$ & $0.829 \pm 0.036$ \\
\hline & Connectome & $82.3 \pm 3.29$ & $80.9 \pm 3.14$ & $84.3 \pm 6.64$ & $0.867 \pm 0.043$ \\
\hline \multirow{3}{*}{ Random forest } & ROI uptake & $70.8 \pm 4.73$ & $81.1 \pm 3.75$ & $59.3 \pm 6.23$ & $0.725 \pm 0.045$ \\
\hline & MCI pattern & $73.1 \pm 4.02$ & $85.4 \pm 2.86$ & $61.4 \pm 8.84$ & $0.787 \pm 0.032$ \\
\hline & Connectome & $76.2 \pm 3.19$ & $87.6 \pm 2.99$ & $62.9 \pm 7.48$ & $0.807 \pm 0.031$ \\
\hline
\end{tabular}

The predictive performance of MCI participants was not involved in the training dataset.

$88.1 \%, 81.2 \%$, and 0.933 , respectively. Moreover, the CPM method achieved the lowest false-positive rate $(18.8 \pm 4.28 \%, 15.7 \pm 6.64 \%$, and $37.1 \pm 7.48 \%)$ and falsenegative rate $(11.9 \pm 3.17 \%, 19.1 \pm 3.14 \%$, and $12.4 \pm 2.99$ $\%)$ in the three classifiers, respectively (Table 3). The receiver-operating-characteristic curves showed a high ability to diagnose MCI groups for metabolic CPM method (AUC, 0.933) but the lower discriminative ability for MCI pattern (AUC, 0.829) and ROI uptake (AUC, 0.831). The result of LR model showed that the information in the metabolic data has been extracted effectively and the goodness of the model fitting is high $\left(X^{2}=7.25\right.$, $P=0.51)$. Besides, the SVM classifier had better diagnostic ability than other classifiers (LR and RF) for the prediction of MCI conversion.

3.3. Discriminative Brain Regions Associated with MCI Conversion. We applied the concept of hubs into the brain regions associated with the progression from MCI to $\mathrm{AD}$ (Table 4). The result of hubs showed that some regions had more discriminative connectivity, including the precentral gyrus, precuneus, lingual gyrus, inferior temporal gyrus, and inferior frontal gyrus (Figures $4(\mathrm{a})-4(\mathrm{c})$ ). When the PET images of pMCI participants were compared with sMCI participant's images, we observed significant hypometabolism in the precuneus, posterior cingulate, superior temporal gyrus, inferior frontal gyrus, etc. The result of metabolic CPM was implied that the hubs were statistically related to the conversion from $\mathrm{MCI}$ to $\mathrm{AD}$.

\section{Discussion}

The accurate and sensitive diagnosis of MCI conversion is a paramount challenge to guide MCI patients for suitable clinical treatments as soon as possible. To address the challenge, in this study, we develop an efficiently metabolic CPM approach to diagnose whether MCI patients will progress to $\mathrm{AD}$ using metabolic images $\left({ }^{18} \mathrm{~F}\right.$-FDG-PET). The performance of our approach suggests that the metabolic connectivity derived by connectome analysis could be used for
TABle 3: Predictive performance of different methods among MCI groups.

\begin{tabular}{lccc}
\hline Classifier & Method & FPR & FNR \\
\hline \multirow{3}{*}{ SVM } & ROI uptake & $33.3 \pm 2.51$ & $17.8 \pm 1.72$ \\
& MCI pattern & $32.9 \pm 6.29$ & $16.3 \pm 4.46$ \\
& Connectome & $18.8 \pm 4.28$ & $11.9 \pm 3.17$ \\
\hline \multirow{3}{*}{ LR model } & ROI uptake & $39.3 \pm 4.83$ & $18.9 \pm 5.99$ \\
& MCI pattern & $33.2 \pm 5.91$ & $17.7 \pm 2.49$ \\
& Connectome & $15.7 \pm 6.64$ & $19.1 \pm 3.14$ \\
\hline \multirow{3}{*}{ Random forest } & ROI uptake & $40.7 \pm 6.23$ & $18.9 \pm 3.75$ \\
& MCI pattern & $38.6 \pm 8.84$ & $14.6 \pm 2.86$ \\
& Connectome & $37.1 \pm 7.48$ & $12.4 \pm 2.99$ \\
\hline
\end{tabular}

TABLE 4: The information of brain regions associated with MCI conversion.

\begin{tabular}{lcccc}
\hline \multirow{2}{*}{ Brain labels } & Region & \multicolumn{3}{c}{ MNI coordinate } \\
& & $X$ & $Y$ & $Z$ \\
\hline 1 & Precentral gyrus (left) & -38.65 & -5.68 & 50.94 \\
11 & Inferior frontal gyrus (left) & -48.43 & 12.73 & 19.02 \\
48 & Lingual gyrus (right) & 16.29 & -66.93 & -3.87 \\
67 & Precuneus (left) & 9.98 & -56.05 & 43.77 \\
89 & Inferior temporal gyrus (left) & -49.77 & 28.05 & -23.17 \\
\hline
\end{tabular}

MCI conversion diagnosis and obtain excellent accuracy compared to other predictive models.

To further reveal the accuracy of our method, we compared the results between previous similar predictive methods and our proposed method, as shown in Table 5 [24-29]. Although the sample sizes and methodology of these studies are not identical, our proposed CPM method has better predictive performances with a large sample size. Maybe because of the deficiency of unbalanced sample sizes, the false-positive rates (FPR) and false-negative rates (FNR) of other methods are significantly higher than that of the 


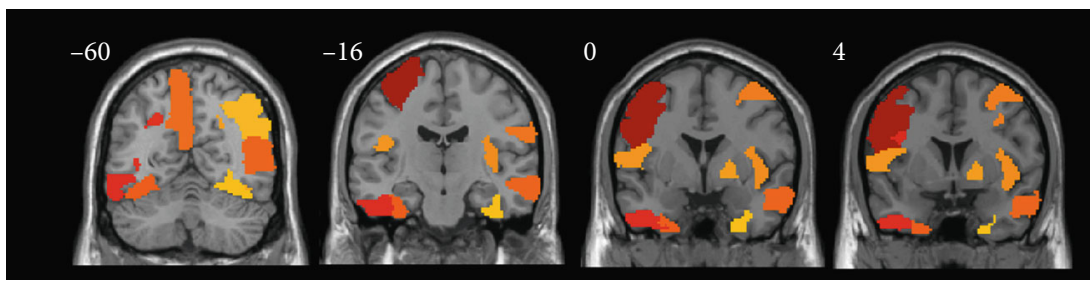

(a)

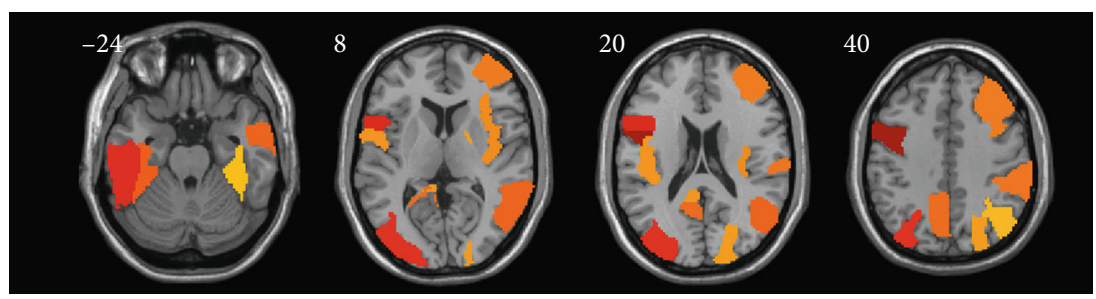

(b)

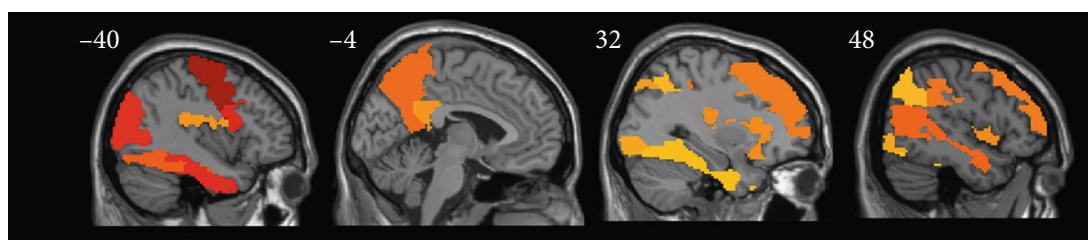

(c)

FIGURE 4: Topographic representations of connectome approach. The hubs regions were acquired from the training dataset using the LASSO approach in 100 iterations and were associated with the conversion from MCI to AD. The overlays are depicted in neurological coronal (a), transverse (b), and sagittal (c) orientations, respectively. Coordinates are displayed in MNI standard space.

TABLE 5: The predictive performance of the different methods in MCI conversion study.

\begin{tabular}{|c|c|c|c|c|c|c|c|c|}
\hline Reference & Method & $\begin{array}{l}\text { Conversion time } \\
\text { (month) }\end{array}$ & $\begin{array}{l}\text { sMCI } \\
(n)\end{array}$ & $\begin{array}{l}\mathrm{pMCI} \\
(n)\end{array}$ & $\begin{array}{c}\text { Accuracy } \\
(\%)\end{array}$ & $\begin{array}{c}\text { Sensitivity } \\
(\%)\end{array}$ & $\begin{array}{c}\text { Specificity } \\
(\%)\end{array}$ & AUC \\
\hline Young et al. & Gaussian process & 36 & 96 & 47 & 65.0 & 66.0 & 64.6 & 0.767 \\
\hline Liu et al. & $\begin{array}{l}\text { Independent component analysis and } \\
\text { Cox model }\end{array}$ & 36 & 108 & 126 & 68.8 & 57.1 & 82.4 & 0.736 \\
\hline Lange et al. & SPM $t$-test & 36 & 77 & 31 & l & l & l & 0.832 \\
\hline Kengo et al. & Logistic regression & 24 & 47 & 41 & 83 & 70 & 90 & 1 \\
\hline Lu et al. & Deep neural network & 36 & 409 & 217 & 81.5 & 78.2 & 82.5 & I \\
\hline Pagani et al. & Independent components analysis & 60 & 27 & 95 & 83.5 & 83.2 & 85.2 & 0.894 \\
\hline Proposed & Metabolic CPM & 36 & 242 & 178 & 85.2 & 88.1 & 86.4 & 0.933 \\
\hline
\end{tabular}

results of our CPM model. Besides, previous studies summarize the MCI conversion approaches and propose a classification framework for reproducible and objective experiments using the ADNI database and other publicly available databases $[13,30]$. The results show that the accuracy of these previous methods is ranging between $62 \%$ and $81 \%$ and the mean accuracy is $74 \%$, and the reproducibility of these methods is not great. Meanwhile, our CPM method underwent a rigorous evaluation strategy to verify its feasibility and reproducibility. This method could capture more detailed and straightforward metabolic information within paired regions which performed as good as or better than many existing approaches. Thus, we believe that our metabolic CPM approach is more effective in predicting MCI conversion.
As an effective tool in the brain neuroscience field, CPM in $\mathrm{AD}$ has been pursued to develop an efficient biomarker for early diagnosis [14]. From the perspective of methodology, it is worth noting that the CPM approach is a generalizable model that takes brain connectivity data as input and generates predictions of MCI progression in novel subjects. In the neurodegenerative progress of $\mathrm{AD}$, the brain changes involve the interaction of many brain regions rather than isolated regions, and neuronal degeneration is associated with cognitive deterioration. The between-region metabolic activities are impaired in MCI patients who are converting to $\mathrm{AD}$. Thus, the most relevant indicator is the identification of corresponding brain regions and their connectivity. The brain network can delineate the full metabolic connectome and the connectivity dynamics of brain metabolism, and these 
findings provide opportunities to develop more accurate predictive models. The progressive disintegration of MCI patients is disclosed by the metabolic network. Therefore, the model based on selected connectivity has excellent diagnostic utility and reveals local brain pathologies.

From the results of connectome analysis, the metabolic connectivity abnormalities in MCI patients who converted to $\mathrm{AD}$ are mainly located in the precentral gyrus, precuneus, lingual, and inferior frontal gyrus. These brain regions are hubs of metabolic connectivity corresponding to synaptic disconnection. Metabolic connectivity in these regions decreases in progressive MCI patients compared to that in stable MCI patients. Previous MCI conversion investigation had shown the metabolic AD conversion-related pattern (ADCRP) which was characterized by relative decreases in temporoparietal, frontal, posterior cingulate, and precuneus cortex between sMCI and pMCI patients, and these results agreed with our experimental findings [22]. The voxel-wise two-sample $t$-test SPM analysis also found a highly similar metabolic difference which verified the pathophysiologic significance of regions that derived our approach [7, 31]. This result also indicates that our proposed metabolic CPM is an effective biomarker for MCI progression prediction.

\section{Conclusion}

In this study, we have proposed an innovative metabolic CPM method based on FDG-PET imaging, which can accurately diagnose whether the patients with MCI will eventually progress to $\mathrm{AD}$. The experiment results suggest that metabolic connectivity can identify the metabolic abnormalities features and abnormal brain regions associated with MCI conversion. Our proposed metabolic CPM approach may be a potential tool with other clinical information to develop biomarkers for predicting the conversion of MCI patients.

\section{Data Availability}

The PET imaging data used to support the findings of this study may be released upon application to the ADNI database (http://adni.loni.usc.edu/).

\section{Conflicts of Interest}

The authors declare that they have no conflicts of interest.

\section{Acknowledgments}

This study was supported in part by the National Key Research and Development Program of China under Grant No. 2018YFC1707704. Data collection and sharing for this project was funded by the Alzheimer's Disease Neuroimaging Initiative (ADNI) (National Institutes of Health Grant U01 AG024904) and DOD ADNI (Department of Defense award number W81XWH-12-2-0012). ADNI is funded by the National Institute on Aging, the National Institute of Biomedical Imaging and Bioengineering, and through generous contributions from the following: AbbVie, Alzheimer's Association; Alzheimer's Drug Discovery Foundation; Araclon
Biotech; BioClinica, Inc; Biogen; Bristol-Myers Squibb Company; CereSpir, Inc; Cogstate; Eisai Inc; Elan Pharmaceuticals, Inc; Eli Lilly and Company; EuroImmun; F. Hoffmann-La Roche Ltd and its affiliated company Genentech, Inc; Fujirebio; GE Healthcare; IXICO Ltd; Janssen Alzheimer Immunotherapy Research \& Development, LLC; Johnson \& Johnson Pharmaceutical Research \& Development LLC; Lumosity; Lundbeck; Merck \& Co., Inc; Meso Scale Diagnostics, LLC; NeuroRx Research; Neurotrack Technologies; Novartis Pharmaceuticals Corporation; Pfizer Inc; Piramal Imaging; Servier; Takeda Pharmaceutical Company; and Transition Therapeutics. The Canadian Institutes of Health Research is providing funds to support ADNI clinical sites in Canada. Private sector contributions are facilitated by the Foundation for the National Institutes of Health (https://www.fnih.org). The grantee organization is the Northern California Institute for Research and Education, and the study is coordinated by the Alzheimer's Therapeutic Research Institute at the University of Southern California. ADNI data are disseminated by the Laboratory for Neuro Imaging at the University of Southern California.

\section{References}

[1] Alzheimer's Association, "2019 Alzheimer's disease facts and figures," Alzheimer's \& Dementia, vol. 15, no. 3, pp. 321-387, 2019.

[2] B. Dubois, H. Hampel, H. H. Feldman et al., "Preclinical Alzheimer's disease: definition, natural history, and diagnostic criteria," Alzheimer's \& Dementia, vol. 12, no. 3, pp. 292-323, 2016.

[3] B. Olsson, R. Lautner, U. Andreasson et al., "Csf and blood biomarkers for the diagnosis of Alzheimer's disease: a systematic review and meta-analysis," The Lancet Neurology, vol. 15, no. 7, pp. 673-684, 2016.

[4] K. Ong, V. L. Villemagne, A. Bahar-Fuchs et al., "18 F-florbetaben $\mathrm{A} \beta$ imaging in mild cognitive impairment," Alzheimer's Research \& Therapy, vol. 5, no. 1, 2013.

[5] L. F. Kalheim, P. Selnes, A. Bjørnerud, C. Coello, K. Vegge, and T. Fladby, "Amyloid dysmetabolism relates to reduced glucose uptake in white matter hyperintensities," Frontiers in Neurology, vol. 7, 2016.

[6] C. Cabral, P. M. Morgado, D. C. Costa, M. Silveira, and For the Alzheimer's Disease Neuroimaging Initiative, "Predicting conversion from mci to ad with FDG-PET brain images at different prodromal stages," Computers in Biology and Medicine, vol. 58, pp. 101-109, 2015.

[7] M. Pagani, F. Nobili, S. Morbelli et al., "Early identification of mci converting to ad: a FDG PET study," European Journal of Nuclear Medicine and Molecular Imaging, vol. 44, no. 12, pp. 2042-2052, 2017.

[8] H. Zhou, J. Jiang, J. Lu et al., "Dual-model radiomic biomarkers predict development of mild cognitive impairment progression to Alzheimer's disease," Frontiers in Neuroscience, vol. 12, 2019.

[9] F. Carbonell, A. Charil, A. P. Zijdenbos, A. C. Evans, B. J. Bedell, and for the Alzheimer's Disease Neuroimaging Initiative, "Hierarchical multivariate covariance analysis of metabolic connectivity," Journal of Cerebral Blood Flow \& Metabolism, vol. 34, no. 12, pp. 1936-1943, 2014. 
[10] M. Liu, D. Cheng, W. Yan, and Alzheimer's Disease Neuroimaging Initiative, "Classification of Alzheimer's disease by combination of convolutional and recurrent neural networks using FDG-PET images," Frontiers in Neuroinformatics, vol. 12, 2018.

[11] H. I. Suk, S. W. Lee, D. Shen, and Alzheimer's Disease Neuroimaging Initiative, "Hierarchical feature representation and multimodal fusion with deep learning for ad/mci diagnosis," NeuroImage, vol. 101, pp. 569-582, 2014.

[12] H. Choi and K. H. Jin, "Predicting cognitive decline with deep learning of brain metabolism and amyloid imaging," Behavioural Brain Research, vol. 344, pp. 103-109, 2018.

[13] N. Smailagic, L. Lafortune, S. Kelly, C. Hyde, and C. Brayne, ${ }^{* 18}$ F-FDG PET for Prediction of Conversion to Alzheimer's Disease Dementia in People with Mild Cognitive Impairment: An Updated Systematic Review of Test Accuracy," Journal of Alzheimer's Disease, vol. 64, no. 4, pp. 1175-1194, 2018.

[14] X. Shen, E. S. Finn, D. Scheinost et al., "Using connectomebased predictive modeling to predict individual behavior from brain connectivity," Nature Protocols, vol. 12, no. 3, pp. 506$518,2017$.

[15] C. J. Stam, "Modern network science of neurological disorders," Nature Reviews. Neuroscience, vol. 15, no. 10, pp. 683695, 2014.

[16] M. N. Hallquist and F. G. Hillary, "Graph theory approaches to functional network organization in brain disorders: a critique for a brave new small-world," Network Neuroscience, vol. 3, no. 1, pp. 1-26, 2019.

[17] T. Yan, W. Wang, L. Yang, K. Chen, R. Chen, and Y. Han, "Rich club disturbances of the human connectome from subjective cognitive decline to Alzheimer's disease," Theranostics, vol. 8, no. 12, pp. 3237-3255, 2018.

[18] W. Jagust, "Imaging the evolution and pathophysiology of Alzheimer disease," Nature Reviews Neuroscience, vol. 19, no. 11, pp. 687-700, 2018.

[19] N. Tzourio-Mazoyer, B. Landeau, D. Papathanassiou et al., "Automated anatomical labeling of activations in spm using a macroscopic anatomical parcellation of the mni mri singlesubject brain," NeuroImage, vol. 15, no. 1, pp. 273-289, 2002.

[20] M. Wang, Alzheimer's Disease Neuroimaging Initiative, J. Jiang et al., "Individual brain metabolic connectome indicator based on Kullback-Leibler divergence similarity estimation predicts progression from mild cognitive impairment to Alzheimer's dementia," European Journal of Nuclear Medicine and Molecular Imaging, 2020.

[21] B. M. Tijms, P. Series, D. J. Willshaw, and S. M. Lawrie, "Similarity-based extraction of individual networks from gray matter MRI scans," Cerebral Cortex, vol. 22, no. 7, pp. 1530-1541, 2012.

[22] G. Blazhenets, Y. Ma, A. Sörensen et al., "Principal component analysis of brain metabolism predicts development of Alzheimer's dementia," Journal of Nuclear Medicine, vol. 60, no. 6, pp. 837-843, 2019.

[23] R. L. Buckner, J. Sepulcre, T. Talukdar et al., "Cortical hubs revealed by intrinsic functional connectivity: mapping, assessment of stability, and relation to Alzheimer's disease," The Journal of Neuroscience, vol. 29, no. 6, pp. 1860-1873, 2009.

[24] J. Young, M. Modat, M. J. Cardoso et al., “Accurate multimodal probabilistic prediction of conversion to Alzheimer's disease in patients with mild cognitive impairment," NeuroImage: Clinical, vol. 2, pp. 735-745, 2013.
[25] K. Liu, K. Chen, L. Yao, and X. Guo, "Prediction of mild cognitive impairment conversion using a combination of independent component analysis and the Cox model," Frontiers in Human Neuroscience, vol. 11, 2017.

[26] C. Lange, P. Suppa, L. Frings, W. Brenner, L. Spies, and R. Buchert, "Optimization of statistical single subject analysis of brain FDG PET for the prognosis of mild cognitive impairment-to-Alzheimer's disease conversion," Journal of Alzheimer's Disease, vol. 49, no. 4, pp. 945-959, 2016.

[27] K. Ito, H. Fukuyama, M. Senda et al., "Prediction of outcomes in mild cognitive impairment by using 18f-FDG-PET: a multicenter study," Journal of Alzheimer's Disease, vol. 45, no. 2, pp. 543-552, 2015.

[28] D. Lu, Alzheimer's Disease Neuroimaging Initiative, K. Popuri, G. W. Ding, R. Balachandar, and M. F. Beg, "Multimodal and multiscale deep neural networks for the early diagnosis of Alzheimer's disease using structural $\mathrm{mr}$ and FDG-PET images," Scientific Reports, vol. 8, no. 1, 2018.

[29] M. Pagani, A. Giuliani, J. Öberg et al., "Progressive Disintegration of Brain Networking from Normal Aging to Alzheimer Disease: Analysis of Independent Components of ${ }^{18} \mathrm{~F}-\mathrm{FDG}$ PET Data," Journal of Nuclear Medicine, vol. 58, no. 7, pp. 1132-1139, 2017.

[30] J. Samper-González, N. Burgos, S. Bottani et al., "Reproducible evaluation of classification methods in Alzheimer's disease: framework and application to mri and pet data," NeuroImage, vol. 183, pp. 504-521, 2018.

[31] R. C. Petersen, E. S. Lundt, T. M. Therneau et al., "Predicting progression to mild cognitive impairment," Annals of Neurology, vol. 85, no. 1, pp. 155-160, 2019. 